\title{
Factors Associated with Noncarious Cervical Lesions in Different Age Ranges: A Cross-sectional Study
} \author{
Anna Thereza Thomé Leão ${ }^{2}$ \\ ${ }^{1}$ Department of Preventive Dentistry, Brazilian Navy, Odontoclínica \\ Central da Marinha, Rio de Janeiro, Brazil \\ ${ }^{2}$ Division of Periodontics, Department of Dental Clinic, Dental \\ School, Universidade Federal do Rio de Janeiro, Rio de Janeiro, \\ Brazil \\ ${ }^{3}$ Division of Restorative Dentistry, Department of Dental Clinic, \\ Brazilian Navy, Odontoclínica Central da Marinha, Rio de Janeiro, \\ Brazil \\ ${ }^{4}$ Division of Periodontics, Department of Dental Clinic, Brazilian \\ Navy, Odontoclínica Central da Marinha, Rio de Janeiro, Brazil \\ ${ }^{5}$ Department of Social and Preventive Dentistry, School of \\ Dentistry, Federal University of Minas Gerais, Belo Horizonte, Brazil
}

Daniela Cia Penoni ${ }^{1,2}$ Maria Elisa da Silva Nunes Gomes Miranda ${ }^{3}$ Flávia Sader ${ }^{4}$

\begin{abstract}
Address for correspondence Daniela Cia Penoni, DDS, MSc, PhD, $1^{\circ}$ Distrito Naval, Odontoclínica Central da Marinha, Praça Barão de Ladário 1, 20091-000 RJ, Brazil (e-mail: ciapenoni@gmail.com).
\end{abstract}

Eur J Dent 2021;15:325-331

\begin{abstract}
Keywords

- tooth wear

- abrasion

- dental

- erosion

- prevalence

Objectives Understanding the possible risk factors of noncarious cervical lesion (NCCL) is important for prevention and clinical management of the condition. The aim of this study was to investigate the factors associated with the prevalence of $\mathrm{NCCL}$ among adolescents, adults, and elderly people.

Materials and Methods A cross-sectional study involving 501 participants aged 15 years or older was conducted. Participants were examined to assess the number of natural teeth and the prevalence and severity of NCCL by calibrated examiners. Data on age, gender, harmful toothbrushing habits, and acidogenic diet were collected through individual interviews. Multivariate Poisson's regression models were used to evaluate the association between the independent variables and the prevalence of NCCL according to the three age groups: 15 to 39,40 to 64 , and 65 years or older.

Results The prevalence of NCCL among participants was 62.5\% (95\% confidence interval: 58.2-66.7). Among 15- to 39-year-old participants, the mean of NCCL was higher in males, those with lower number of teeth and acidogenic diet intake. Males aged 40 to 64 years and those with harmful brushing habits were more likely to present higher mean of NCCL. Elderly people with harmful toothbrushing habits had a greater mean of NCCL.

Conclusion Demographic (age and gender), clinical (number of teeth), and behavioral characteristics (harmful brushing habits and acidogenic diet) were meaningful factors associated with NCCL severity. The above-mentioned relationships varied between age groups.
\end{abstract}

published online

February 3, 2021
DOI https://doi.org/

$10.1055 / \mathrm{s}-0040-1722092$

ISSN 1305-7456. (c) 2021. European Journal of Dentistry.

This is an open access article published by Thieme under the terms of the Creative Commons Attribution-NonDerivative-NonCommercial-License, permitting copying and reproduction so long as the original work is given appropriate credit. Contents may not be used for commercial purposes, or adapted, remixed, transformed or built upon. (https://creativecommons.org/licenses/by-nc-nd/4.0/) Thieme Medical and Scientific Publishers Pvt. Ltd., A-12, 2nd Floor, Sector 2, Noida-201301 UP, India 


\section{Introduction}

Noncarious cervical lesion (NCCL) is clinical conditions characterized by the loss of dental tissue around the cementenamel junction (CEJ) that are not associated with dental caries. ${ }^{1,2}$ Overall, NCCL is a multifactorial dental condition that can affect up to $60 \%$ of the population, ${ }^{2}$ and may vary between age groups and between different geographical areas. ${ }^{1,-6}$ The global prevalence of NCCL was estimated as $46.7 \%$, with greater occurrence among people older than 30 years $(54.1 \%){ }^{7}$ NCCLs may result in dentin hypersensitivity, pulpal disorders and can compromise aesthetics. ${ }^{8}$ Severe cases of NCCLs are also a risk factor of dental fractures. NCCL is the second leading cause of dental restoration in permanent teeth. ${ }^{9}$

The enamel in cervical region is aprismatic and thinner than in other areas of the tooth, which increases the susceptibility to mineral losses. Biocorrosion and abrasion (tooth wear) are some of the proposed mechanisms for the initiation and development of NCCL. ${ }^{10}$ The co-occurrence of these factors can also accelerate the development of NCCLs. ${ }^{10}$ The biocorrosion mechanism related to NCCL occurs through the action of endogenous and exogenous acids that cause the demineralization of the dental tissues. ${ }^{11-13}$ Endogenous acids are produced and released from the stomach and reach the mouth as a result of gastroesophageal reflux disease or eating desorders, ${ }^{14}$ whereas exogenous acids affect dental tissues through acidogenic diet ${ }^{15,16}$ or work-related environmental exposures. ${ }^{17}$ Dietary habits are important factors for dental erosion, whereas acidogenic drinks and alcoholic beverages intake are related to erosive tooth wear., ${ }^{2,18}$

Another cofactor that can act synergistically on the induction and development of NCCLs is the stress concentration. ${ }^{10}$ Finite element analysis has demonstrated that the tooth cervical region is the zone where higher levels of stress occur commonly. Occlusal loading forces can cause microcracks along the CEJ and, consequently, microstructural loss of tissue in this region. ${ }^{10,19}$

Excessive force during toothbrushing and use of hard toothbrushes can contribute to the occurrence of NCCLs. ${ }^{20}$ Toothbrush abrasion may accelerate the initiation and exacerbation of NCCLs when combined with the consumption of acidogenic diet, including the intake of citrus fruit juice, carbonated drinks, wine, and vinegar intake. ${ }^{21-23}$

The progression of NCCLs is a time-dependent process resulting from the accumulative exposure of the etiological factors throughout the lifespan. The interaction of various mechanisms, such as stress, friction, and biocorrosion, can indicate the complex etiology of these multifactorial lesions. ${ }^{10}$ This suggests the prevalence of NCCLs is higher in older people than in younger ones. ${ }^{5,24,25}$ NCCLs can seriously impact on person's quality of life related to oral health. Oral health education provided by qualified professionals might prevent the occurrence of NCCL. ${ }^{26}$

The multifactorial etiology of NCCLs involves the combined effects of possible risk factors, including gender, acidic diet, force during toothbrushing, and age..$^{27.28}$ Although some characteristics have been associated with the prevalence of NCCL, there is a lack of studies accessing the association between these factors with the extension of NCCLs in different age groups.

The adequate prevention and management of NCCLs treatment involve the understanding of the associated factors of NCCLs. Therefore, the aim of this study was to investigate the association of age, gender, harmful toothbrushing habits, and acidogenic diet with the prevalence of NCCLs. In addition, the aforementioned relationship was investigated according to the three age groups: 15 to 39 years (younger adults), 40 to 64 years (adults), and 65 years or older (elderly people).

\section{Materials and Methods}

\section{Study Design and Setting}

This cross-sectional study involved a convenience sample of 501 patients registered at the Naval Dental Centre (Odontoclínica Central da Marinha/OCM), Rio de Janeiro, Brazil in 2018 and 2019. The OCM is a military institution of the Brazilian Navy that provides primary and specialized dental care, including dental health education, periodontal therapy, and restorative procedures. Militaries on active duty, pensioners, and their dependent aged 12 years or older are entitled for dental care and treatment at OCM.

\section{Participants}

All the patients who attended the division of restorative dentistry for the first time in March and September 2018 and 2019 were invited to participate in the study, irrespective of gender and age. Patients who had already been treated in the division of restorative dentistry were excluded.

This study was approved by the research ethics committee of the Hospital Naval Marcílio Dias, approval number 3.399.461, and it conforms to the ethical principles of the Declaration of Helsinki.

\section{Data Collection and Variables}

Demographic data were age and gender. The acidic diet was a dichotomous variable, that is, "no" $=0$ or "yes" $=1$. When the participant reported a daily intake of acid fruits, or beverages, including sport drinks, fruit juices, or wine, they were classified as (1). If they informed that none of these items was consumed on a daily basis, they were classified as (0). Harmful toothbrushing habits were assessed using the following question: "Do you apply excessive force when brushing your teeth or do you use toothbrush with nonsoft bristles?" The response for harmful toothbrushing habits was "no" $=0$ or "yes" $=1$. Dental clinical data were number of natural teeth and NCCLs.

The participants were clinically examined to assess the number of natural teeth and the number and severity of NCCLs through a full-mouth examination. Dental visual examinations were performed using the light and a plain dental mirror at the dental offices of the OCM. Clinical loss of mineral dental structure at the CEJ in the buccal and/or lingual dental surfaces after visual examination was considered presence of NCCL. The clinical presentation of NCCL could include any morphology that characterized these lesions: 
rounded or concave, wedge shaped, and mixed. Cervical dental surfaces with restorations and those with extensive dental caries and dental fractures were not considered NCCL. Teeth with NCCLs were classified as in need of dental fillings and not in need of dental fillings. NCCLs with a depth higher than $1 \mathrm{~mm}$ were classified in need for dental restoration according to the subjective assessment of the examiner.

\section{Clinical Calibration}

Dental examinations were conducted after training and clinical calibration of 13 examiners with expertise in restorative dentistry. A calibration study involving 15 subjects was conducted to assess interexaminer reliability of NCCLs. The patients were selected at OCM dental clinic and were dentally examined for the presence of NCCL. One experienced dentist (M.E.S.N.G.M.) acted as the gold standard to assess the clinical reliability of the other 12 examiners. Kappa coefficients between examiners ranged from 0.86 to 1.00 .

\section{Data Analysis}

Descriptive data of demographic data, harmful toothbrushing habits, acidogenic diet, and dental clinical measures were presented using proportions and means ( \pm standard deviation $[\mathrm{SD}]$ ) for categorical variables and numeric variables according to the prevalence of NCCL (presence of NCCL $\times$ absence of NCCL). Shapiro-Wilk's test was used to test the distribution of the data. Since the null hypothesis of normality was rejected, nonparametric tests were used. Statistical differences between the groups were evaluated using the chi-square test for categorical variables and Mann-Whitney's test for continuous variables. The level of statistical significance was 0.05 . The number of teeth, NCCLs and NCCLs in need of dental fillings, was also compared between the three age groups: 15 to 39, 40 to 64, and 65 years or older using Kruskal-Wallis' test. Severity of NCCL was evaluated according to the number of teeth affected by NCCL in the individual.
Multivariable Poisson's regression with robust covariance was used to test the association of gender, acidogenic diet, harmful toothbrushing habits, and number of teeth with NCCLs according to the three age groups to estimate rate ratio (RR) and 95\% confidence intervals (Cls).

All data analyses were performed using the SPSS software version 21.0 (Statistical Package for the Social Sciences; SPSS Inc., Chicago, United States).

\section{Results}

Of the 1,989 patients registered at the Naval Dental Centre, 559 were dentally examined at the restorative dentistry clinic between 2018 and 2019. Of them, 501 had complete clinical data. They were aged 15 years or older. The mean age of the sample was $52.5(\mathrm{SD}=17.4)$ and $53.5 \%$ were males. The participants included 116 (23.2\%) adolescents and younger adults aged between 15 and 39 years, 231 (46.1\%) adults aged between 40 and 64 years, and 154 (30.7\%) elderly people aged 65 years or older. The prevalence of at least one tooth with NCCL was $62.5 \%$ (95\% CI: 58.2-66.7). The distribution of sex, age, toothbrushing habits, acidogenic diet, and number of teeth is shown in - Table 1. The prevalence of NCCL was significantly associated with male gender, age, harmful toothbrushing, acidogenic diet, and number of natural teeth.

- Table 2 shows the presents number of teeth, NCCLs and NCCL with interventional need for the three age groups. The mean number of NCCLs was $1.09,3.19$, and 2.50 for the 15 to 39,40 to 64 , and $\geq 65$ years old age groups, respectively. The number of teeth, NCCLs and NCCL in need of dental fillings, was statistically different between the age groups $(p<0.001)$.

The results from crude Poisson's regression analysis showed that the number of NCCL was significantly associated with greater age, male gender, acidogenic diet, and harmful toothbrushing habits for the total sample. Male gender was associated with NCCL among participants aged 40 to 64 years.

Table 1 Descriptive analysis of the sample according to the prevalence of NCCLs

\begin{tabular}{|c|c|c|c|c|}
\hline Variables & $\begin{array}{l}\text { Total sample } \\
(N=501)\end{array}$ & $\begin{array}{l}\text { Absence of } \\
\text { NCCLs }(N=188)\end{array}$ & $\begin{array}{l}\text { Presence of } \\
\text { NCCLs }(N=313)\end{array}$ & $p$-Value \\
\hline Age, median; interquartile range & $53.0 ; 40.0-67.0$ & $42.5 ; 29.0-61.8$ & $57.0 ; 48.0-69.0$ & $<0.001^{\mathrm{a}}$ \\
\hline \multicolumn{5}{|l|}{ Sex, \% } \\
\hline Male & $268(53.5)$ & $83(44.1)$ & $185(59.1)$ & \multirow[t]{2}{*}{$0.001^{\mathrm{b}}$} \\
\hline Female & $233(46.5)$ & 105 (55.9) & $128(40.9)$ & \\
\hline \multicolumn{5}{|l|}{ Acidic diet intake, $N(\%)$} \\
\hline No & $389(77.6)$ & $161(85.6)$ & $228(72.8)$ & \multirow[t]{2}{*}{$<0.001^{\mathrm{b}}$} \\
\hline Yes & $112(22.4)$ & $27(14.4)$ & $85(27.2)$ & \\
\hline Number of teeth, median; interquartile range & $26.0 ; 21.0-28.0$ & $28.0 ; 23.5-29.0$ & $24.0 ; 20.0-28.0$ & $<0.001^{\mathrm{a}}$ \\
\hline \multicolumn{5}{|l|}{ Harmful toothbrushing habits, $\%$} \\
\hline No & $303(60.5)$ & $145(77.1)$ & $158(50.5)$ & \multirow[t]{2}{*}{$<0.001^{b}$} \\
\hline Yes & $198(39.5)$ & $43(22.9)$ & $155(49.5)$ & \\
\hline
\end{tabular}

Abbreviations: NCCLs, noncarious cervical lesions; SD, standard deviation.

Note: Data expressed as median; interquartile range, or absolute number (\%).

aMann-Whitney's test.

${ }^{\mathrm{b} C h i}$-square test. 
Table 2 Number and percentage of NCCLs and NCCLs in need of dental fillings according to the three age groups

\begin{tabular}{|c|c|c|c|c|}
\hline & \multicolumn{3}{|c|}{ Age groups } & \multirow[t]{2}{*}{$p$-Value ${ }^{\text {a }}$} \\
\hline & $15-39 y$ & $40-64$ y & $\geq 65 y$ & \\
\hline \multicolumn{5}{|l|}{ NCCLs } \\
\hline Median; interquartile range & $0.0 ; 0.0-2.0$ & $2.0 ; 0.0-4.5$ & $2.0 ; 0.0-4.0$ & $<0.001$ \\
\hline Percentage & $0.0 ; 0.0-6.3$ & $10.3 ; 0.0-18.8$ & $9.8 ; 0.0-23.5$ & $<0.001$ \\
\hline \multicolumn{5}{|l|}{ NCCLs in need of dental fillings } \\
\hline Median; interquartile range & $0.0 ; 0.0-0.0$ & $0.0 ; 0.0-2.0$ & $1.0 ; 0.0-3.0$ & $<0.001$ \\
\hline Percentage & $0.0 ; 0.0-0.0$ & $0.0 ; 0.0-8.0$ & $6.9 ; 0.0-16.7$ & $<0.001$ \\
\hline
\end{tabular}

Abbreviation: NCCLs, noncarious cervical lesions.

Note: Data expressed as median and interquartile range.

${ }^{a} p$-Values refer to the comparison between the three age groups using Kruskal-Wallis' test.

Table 3 Crude associations of age, gender, harmful toothbrushing habits, and acidogenic diet with the number of noncarious cervical lesions according to the three age groups

\begin{tabular}{|c|c|c|c|}
\hline \multirow[t]{2}{*}{ Variables } & $\begin{array}{l}15-39 y \\
(N=116)\end{array}$ & $\begin{array}{l}40-64 \text { y } \\
(N=231)\end{array}$ & $\begin{array}{l}\geq 65 y \\
(N=154)\end{array}$ \\
\hline & RR (95\% CI) & RR (95\% CI) & RR (95\% CI) \\
\hline Number of teeth & $0.88(0.74-1.05)$ & $1.01(0.99-1.04)$ & $1.02(0.99-1.04)$ \\
\hline \multicolumn{4}{|l|}{ Gender } \\
\hline Female & 1 & 1 & 1 \\
\hline Male & $2.05(0.98-4.31)$ & $1.66(1.27-2.16)^{\mathrm{a}}$ & $1.24(0.86-1.77)$ \\
\hline \multicolumn{4}{|l|}{ Acidogenic diet } \\
\hline No & 1 & 1 & 1 \\
\hline Yes & $2.56(1.34-4.88)^{\mathrm{a}}$ & $1.56(1.20-2.02)^{a}$ & $1.37(0.93-2.04)$ \\
\hline \multicolumn{4}{|c|}{ Harmful tooth brushing habits } \\
\hline No & 1 & 1 & 1 \\
\hline Yes & $1.88(0.96-3.72)$ & $1.75(1.35-2.26)^{\mathrm{a}}$ & $1.51(1.07-2.13)^{\mathrm{b}}$ \\
\hline
\end{tabular}

Abbreviations: $\mathrm{Cl}$, confidence interval; $\mathrm{RR}$, rate ratio.

${ }^{\mathrm{a}} \mathrm{p} \leq 0.01$.

${ }^{\mathrm{b}} \mathrm{p} \leq 0.05$.

Acidogenic diet was associated with NCCL in the 15 to 39 and 40 to 64 years old age groups. Harmful toothbrushing habits were associated with NCCL in participants aged 40 to 64 and $\geq$ 65 years ( - Table 3 ).

Number of teeth, gender, acidogenic diet, and harmful toothbrushing habits remained statistically associated with NCCL in the adjusted Poisson's regression analysis (-Table 4). Male gender increased the mean of NCCL in the 15 to 39 years old (RR: 2.64; 95\% CI: $1.35-5.18$ ) and 40 to 64 years old (RR: 1.59 ; 95\% CI: 1.21-2.09) age groups. Patients aged 15 to 39 years reporting acidogenic diet were 2.29 times more likely to have a higher mean of NCCL than those who did not report acidogenic diet. In addition, each missing tooth represented an increase of $17 \%$ on the mean of NCCL (RR: 0.83 ; 95\% CI: 0.69-0.99). The fewer the number of teeth, the greater the extent of NCCLs in this age group. The mean prevalence of NCCL of patients reporting harmful toothbrushing was 52\% (95\% CI: $1.17-1.99$ ) and $43 \%$ (95\% CI: 1.01-2.03) higher in the age groups 40 to 64 and 65 years or older, respectively, than those not reporting harmful toothbrushing (-Table 4 ).

\section{Discussion}

The findings of this study suggest that greater age, male gender, harmful toothbrushing habits, acidogenic diet, and number of teeth are associated factors with NCCLs in individuals aged 15 years or older. Although these results refer to the studied population as a whole, the above-mentioned relationships varied between the different age groups investigated. Male gender was associated with NCCL among patients aged 15 to 39 and 40 to 64 years, but not among elderly people aged 65 years or older. Patients aged 15 to 39 years reporting acidogenic diet were more likely to have a higher number of NCCL compared with those who did not report acidogenic diet. Acidogenic diet and number of teeth were associated with NCCL only in patients aged 15 to 39 years. On the contrary, harmful toothbrushing was associated with NCCL in the 40 to 64 and 65 years or older age groups. These findings may indicate that acidogenic diet is a relevant aspect related to NCCL in adolescents and younger adults, whereas harmful toothbrushing is more important for the occurrence of NCCL in older age groups. 
Table 4 Multivariable Poisson's regression on the association of age, gender, harmful toothbrushing, and acidogenic diet with the number of noncarious cervical lesions according to the three age groups

\begin{tabular}{|c|c|c|c|}
\hline \multirow[t]{2}{*}{ Variables } & $\begin{array}{l}15-39 y \\
(N=116)\end{array}$ & $\begin{array}{l}40-64 y \\
(N=231)\end{array}$ & $\begin{array}{l}\geq 65 y \\
(N=154)\end{array}$ \\
\hline & RR (95\% Cl) & RR $(95 \% \mathrm{CI})$ & $\operatorname{RR}(95 \% \mathrm{Cl})$ \\
\hline Number of teeth & $0.83(0.69-0.99)^{\mathrm{a}}$ & $1.01(0.98-1.03)$ & $1.01(0.99-1.04)$ \\
\hline \multicolumn{4}{|l|}{ Gender } \\
\hline Female & 1 & 1 & 1 \\
\hline Male & $2.64(1.35-5.18)^{b}$ & $1.59(1.21-2.09)^{\mathrm{b}}$ & $1.20(0.85-1.70)$ \\
\hline \multicolumn{4}{|l|}{ Acidogenic diet } \\
\hline No & 1 & 1 & 1 \\
\hline Yes & $2.29(1.20-4.35)^{b}$ & $1.28(0.86-1.90)$ & $1.28(0.85-1.93)$ \\
\hline \multicolumn{4}{|c|}{ Harmful tooth brushing habits } \\
\hline No & 1 & 1 & 1 \\
\hline Yes & $1.82(0.92-3.59)$ & $1.52(1.17-1.99)^{\mathrm{b}}$ & $1.43(1.01-2.03)^{\mathrm{a}}$ \\
\hline
\end{tabular}

Abbreviations: $\mathrm{Cl}$, confidence interval; $\mathrm{RR}$, rate ratio.

${ }^{a} p \leq 0.05$.

${ }^{\mathrm{b}} \mathrm{p} \leq 0.01$.

The overall prevalence of $62.5 \%$ of NCCL is in accordance with other studies. 2,25,27,29 Possible discrepancies on the prevalence among studies may be due to variations in their methodology, such as the inclusion criteria, clinical examination procedures, and variations in case definition of NCCL. Most of previous studies have used clinical tactile and visual examinations, while others have used visual examination only, as ours. ${ }^{17,27,29,30}$ The prevalence of NCCL could also differ if it was based on other indexes, as one that assess NCCL based on wear and extent of hard tissue loss according to the size of the area affected and the depth of the defect. ${ }^{31}$ One area of consensus is the recognition that visual dentin exposure is a reliable indicator of substantial loss of tooth tissue that can also be used as threshold of a dichotomous measure in tooth wear scoring system. ${ }^{32}$

The relationship between greater age and NCCL is in accordance with recent reports. ${ }^{7,29}$ Since higher age means a longer exposure of the teeth to etiological factors of NCCL, elderly people tend to have a higher likelihood to present NCCLs than younger people. ${ }^{1,4,7,27,30}$ Gingival recession and having fewer teeth that results in higher occlusal load, enamel, and dentin microstructural defects are possible factors that may explain the increase of NCCL in older age. ${ }^{29}$ Furthermore, the higher the age, the greater the frequency of NCCL in need of dental fillings. This finding suggests the severity of the NCCL is also greater in older people and highlights the importance of investigating the etiological factors, the development of appropriate preventive strategies, and management of NCCL. ${ }^{30}$

Men were more likely to present more NCCL than women. This finding was observed for the total sample and among those aged 15 to 39 and 40 to 64 years, and is in accordance with other research that showed a higher prevalence of NCCL in males. ${ }^{26,29}$ Males may present more NCCLs than females, mainly in youngers and adults, due to a higher bite force and greater masticatory strength. ${ }^{8,33}$ On the contrary, gender did not predict multiple NCCLs in elderly people possibly because the above-mentioned mechanisms are similar between males and females in this age group.

Harmful toothbrushing habits were associated with greater risk of NCCL in the total sample and among participants aged 40 years or older. This finding has clinical relevance, since harmful toothbrushing habits can be easily assessed and detected by clinician. Therefore, it represents an opportunity for prevention of NCCL through professional advice. Frequency of toothbrushing, excessive force during toothbrushing, as well as toothbrush hardness was shown to act as cofactors in the multifactorial etiology of NCCLs. ${ }^{20}$ The type of toothbrush bristle plays an important role in the development of NCCL. The ordinary/flat trimmed version is more abrasive, while the feathered toothbrush caused less wedge-shaped lesions in the cervical area of the tooth. ${ }^{34}$ Some authors state that the high prevalence of NCCL on the buccal surface of the teeth is due to the influence of harmful toothbrushing habits that should be considered as a predictor for the initiation of NCCLs. ${ }^{25,35}$ Besides, obsessive-compulsive symptoms may be related to a cleaning compulsion, frequency, and the intensity of tooth brushing which may be associated with gingival recession and loss of enamel and dentine. ${ }^{36}$ It has been suggested that the correct use of toothbrushes would only cause minimal dentin wear throughout life. ${ }^{9}$ However, upon root dentin exposure, the use of low-abrasive toothpastes during toothbrushing is advisable to reduce the risk of NCCLs development. ${ }^{37}$ Although toothbrushing habits may not be sufficient to initiate the NCCL, it may increase the progression of an initial NCCL caused by biomechanical factors, such as occlusal alterations and bruxism, ${ }^{25}$ esophageal problems, ${ }^{11}$ acidogenic diet, ${ }^{16,21-23}$ or professional exposition to acid mists. ${ }^{17}$ Inadequate toothbrushing may harm eroded surfaces by removing the demineralized enamel surface layer. The in vivo effects of toothbrushing on eroded dentine are not fully elucidated. ${ }^{20}$ The link between higher number of NCCL 
and harmful toothbrushing habits among participants aged 40 years or older observed in this study may suggest that this behavior may act through lifespan. ${ }^{25}$ Harmful toothbrushing habits may promote the progression of NCCL that have been initiated as a result of other risk factors, such as acidogenic diet, during adolescence, and early adulthood.

The mean of NCCL was significantly higher among patients aged 15 to 39 years who reported acidogenic diet than those who did not report this type of diet. Therefore, the type of diet seems an important behavior that should be addressed by dental professionals. The consumption of acidogenic diet among young people deserves attention due to the following reasons: young people who have healthy life tend to have a diet rich in acid fruits and to consume whey protein and carbohydrates/energy drinks after physical activities. ${ }^{15}$ On the contrary, they may lead stressful life and experience anxiety that predispose them to gastrointestinal reflux. In such circumstances, these patients may need drugs to reduce gastrointestinal reflux that alter the salivary flow ${ }^{38}$ and increase the risk of losing dental structure. ${ }^{38}$ Besides, soft drinks and alcoholic drinks consumption ${ }^{16}$ have been associated with the development of NCCL by biocorrosion.

The age group 40 to 64 years presented a higher mean number of teeth with NCCL than those aged 65 years or older, although the frequency of NCCL was greater in the latter age group. The number of teeth should be taken into account when investigating the possible risk factors for the prevalence and extent of NCCLs, mainly among younger people, since each missing tooth in this group was linked to an increase in the mean of NCCL. This is possibly due to the higher occlusal load on the remaining teeth. ${ }^{29}$

Several limitations must be considered when interpreting our findings. One of them is the possible measurement bias related to the use of self-reported measures about harmful toothbrushing habits and acidogenic diet. However, there are not standardized methods to evaluate toothbrushing habits and acidogenic diet. ${ }^{5}$ Another limitation is the lack of information on possible etiological factors for NCCLs, including occlusal trauma and bruxism. Data on salivary flow and buffer effect capacity were not obtained since the present study focused on the clinical aspects related to NCCLs. The convenience sample selected in this study was gathered among patients who had sought for dental treatment in the Division of Restorative Dentistry in an institution of the Brazilian Navy. Then, the sample may not represent the general population since all participants had accessed to dental services. Finally, our findings limit the causal inference on the link between harmful toothbrushing habit and acidogenic diet and NCCLs because of the cross-sectional design. Longitudinal studies are necessary to confirm whether these risk indicators predict greater number of NCCLs. Other interesting issues related to NCCL deserve further investigation, including whether harmful toothbrushing habit and acidogenic diet predict hypersensitivity and gingival recession. The role of toothbrushing methods, duration, frequency, and force on the progression of NCCL should also be explored in future studies.

There are some clinical implications associated to the findings of this study. Oral health professionals should be aware of the possible risk factors involved in the etiology and progression of NCCL in adolescents, adults, and elderly people, as well as how they affect NCCL occurrence in different age groups. These lesions may progress if not managed adequately. Progressive NCCL may lead to tooth loss in elderly people due to pulp problems, bacterial contamination, fractures, ${ }^{6}$ or hypersensitivity. ${ }^{39}$ Restoration, periodontal surgery, dietary advice, occlusal adjustment, and other procedures may prevent NCCLs or improve the aesthetics and functional consequences of NCCLs. Regular dental visits involving clinical examination of incipient NCCLs and investigation on the type of diet, health status, and patient's lifestyle are important strategies related to preventive dentistry.

\section{Conclusion}

This study showed that harmful toothbrushing habits, acidogenic diet, male gender, higher age, and having fewer teeth were meaningful predictors for NCCLs. However, the relevance of these possible risk factors on NCCL depends on the age group. In younger people, male gender, acidogenic diet, and fewer teeth were relevant factors of NCCL, whereas male gender and harmful toothbrushing habits were associated with NCCL among those between 40 and 64 years. Among those aged 65 years or older, NCCL was linked to harmful toothbrushing habits.

\section{Conflict of Interest}

None declared.

\section{Acknowledgments}

The authors are grateful to the staff of the Division of Restorative Dentistry of the Naval Dental Center for contributing to data collection.

\section{References}

1 Aw TC, Lepe X, Johnson GH, Mancl L. Characteristics of noncarious cervical lesions: a clinical investigation. J Am Dent Assoc 2002;133(6):725-733

2 Yoshizaki KT, Francisconi-Dos-Rios LF, Sobral MA, Aranha AC, Mendes FM, Scaramucci T. Clinical features and factors associated with non-carious cervical lesions and dentin hypersensitivity. J Oral Rehabil 2017;44(2):112-118

3 Bartlett DW, Shah P. A critical review of non-carious cervical (wear) lesions and the role of abfraction, erosion, and abrasion. J Dent Res 2006;85(4):306-312

4 Borcic J, Anic I, Urek MM, Ferreri S. The prevalence of non-carious cervical lesions in permanent dentition. J Oral Rehabil 2004;31(2):117-123

5 Teixeira DNR, Zeola LF, Machado AC, et al. Relationship between noncarious cervical lesions, cervical dentin hypersensitivity, gingival recession, and associated risk factors: a cross-sectional study. J Dent 2018;76:93-97 
6 Levitch LC, Bader JD, Shugars DA, Heymann HO. Non-carious cervical lesions. J Dent 1994;22(4):195-207

7 Teixeira DNR, Thomas RZ, Soares PV, Cune MS, Gresnigt MMM, Slot DE. Prevalence of noncarious cervical lesions among adults: a systematic review. J Dent 2020;95:103285

8 Grippo JO, Simring M, Schreiner S. Attrition, abrasion, corrosion and abfraction revisited: A new perspective on tooth surface lesions. J Am Dent Assoc 2004;135(8):1109-1118, quiz 1163-1165

9 Shellis RP, Addy M. The interactions between attrition, abrasion and erosion in tooth wear. Monogr Oral Sci 2014;25:32-45

10 Grippo JO, Simring M, Coleman TA. Abfraction, abrasion, biocorrosion, and the enigma of noncarious cervical lesions: a 20-year perspective. J Esthet Restor Dent 2012;24(1):10-23

11 Barron RP, Carmichael RP, Marcon MA, Sàndor GK. Dental erosion in gastroesophageal reflux disease. J Can Dent Assoc 2003;69(2):84-89

12 Bartlett D. Etiology and prevention of acid erosion. Compend Contin Educ Dent 2009;30(9):616-620

13 GrippoJO. Biocorrosion vs. erosion: the 21 st century and a time to change. Compend Contin Educ Dent 2012;33(2):e33-e37

14 Meurman JH, Toskala J, Nuutinen P, Klemetti E. Oral and dental manifestations in gastroesophageal reflux disease. Oral Surg Oral Med Oral Pathol 1994;78(5):583-589

15 Wang X, Lussi A. Functional foods/ingredients on dental erosion. Eur J Nutr 2012;51(Suppl 2) :S39-S48

16 Lussi A, Megert B, Shellis RP, Wang X. Analysis of the erosive effect of different dietary substances and medications. $\mathrm{Br}$ J Nutr 2012;107(2):252-262

17 Bomfim RA, Crosato E, Mazzilli LE, Frias AC.. Prevalence and risk factors of non-carious cervical lesions related to occupational exposure to acid mists. Braz Oral Res 2015;29-29

18 Kitasako Y, Sasaki Y, Takagaki T, Sadr A, Tagami J. Age-specific prevalence of erosive tooth wear by acidic diet and gastroesophageal reflux in Japan. J Dent 2015;43(4):418-423

19 Jakupović S, Anić I, Ajanović M, et al. Biomechanics of cervical tooth region and noncarious cervical lesions of different morphology; three-dimensional finite element analysis. Eur J Dent 2016;10(3):413-418

20 Wiegand A, Burkhard JP, Eggmann F, Attin T. Brushing force of manual and sonic toothbrushes affects dental hard tissue abrasion. Clin Oral Investig 2013;17(3):815-822

21 Kitchens M, Owens BM. Effect of carbonated beverages, coffee, sports and high energy drinks, and bottled water on the in vitro erosion characteristics of dental enamel. J Clin Pediatr Dent 2007;31(3):153-159

22 Wongkhantee S, Patanapiradej V, Maneenut C, Tantbirojn D. Effect of acidic food and drinks on surface hardness of enamel, dentine, and tooth-coloured filling materials. J Dent 2006;34(3):214-220

23 Attin T, Knöfel S, Buchalla W, Tütüncü R. In situevaluation of different remineralization periods to decrease brushing abrasion of demineralized enamel. Caries Res 2001;35(3):216-222
24 Bader JD, Levitch LC, Shugars DA, Heymann HO, McClure F. How dentists classified and treated non-carious cervical lesions. J Am Dent Assoc 1993;124(5):46-54

25 Yang J, Cai D, Wang F, et al. Non-carious cervical lesions (NCCLs) in a random sampling community population and the association of NCCLs with occlusive wear. J Oral Rehabil 2016;43(12):960-966

26 Lai ZY, Zhi QH, Zhou Y, Lin HC. Prevalence of non-carious cervical lesions and associated risk indicators in middle-aged and elderly populations in Southern China. Chin J Dent Res 2015;18(1):41-50

27 Que K, Guo B, Jia Z, Chen Z, Yang J, Gao P. A cross-sectional study: non-carious cervical lesions, cervical dentine hypersensitivity and related risk factors. J Oral Rehabil 2013;40(1):24-32

28 Alvarez-Arenal A, Alvarez-Menendez L, Gonzalez-Gonzalez I, Alvarez-Riesgo JA, Brizuela-Velasco A, deLlanos-Lanchares $\mathrm{H}$. Non-carious cervical lesions and risk factors: a case-control study. J Oral Rehabil 2019;46(1):65-75

29 Kolak V, Pešić D, Melih I, Lalović M, Nikitović A, Jakovljević A. Epidemiological investigation of non-carious cervical lesions and possible etiological factors. J Clin Exp Dent 2018;10(7):e648-e656

30 Ringelberg ML, Gilbert GH, Antonson DE, et al. Root caries and root defects in urban and rural adults: the Florida Dental Care Study. J Am Dent Assoc 1996;127(7):885-891

31 Smith BG, Knight JK. An index for measuring the wear of teeth. Br Dent J 1984;156(12):435-438

32 López-Frías FJ, Castellanos-Cosano L, Martín-González J, LlamasCarreras JM, Segura-Egea JJ. Clinical measurement of tooth wear: Tooth wear indices. J Clin Exp Dent 2012;4(1):e48-e53 doi:10.4317/jced.50592

33 Scudine KGO, Pedroni-Pereira A, Araujo DS, Prado DGA, Rossi AC, Castelo PM. Assessment of the differences in masticatory behavior between male and female adolescents. Physiol Behav 2016;163:115-122

34 Turssi CP, Kelly AB, Hara AT. Toothbrush bristle configuration and brushing load: effect on the development of simulated non-carious cervical lesions. J Dent 2019;86:75-80

35 Khan F, Young WG, Shahabi S, Daley TJ. Dental cervical lesions associated with occlusal erosion and attrition. Aust Dent J 1999;44(3):176-186

36 Mafla AC, Lopez-Moncayo LF. Dentine sensitivity risk factors: a case-control study. Eur J Dent 2016;10(1):1-6

37 Sabrah AH, Turssi CP, Lippert F, Eckert GJ, Kelly AB, Hara AT. 3D-Image analysis of the impact of toothpaste abrasivity on the progression of simulated non-carious cervical lesions. J Dent 2018;73:14-18

38 Zero DT. Etiology of dental erosion-extrinsic factors. Eur J Oral Sci 1996;104(2 (Pt 2)):162-177

39 Exarchou C, Betsani I, Sakellari D, Chatzopoulou D, Gillam D. A survey of dentists in the management of dentine hypersensitivity: a questionnaire-based study. Eur J Dent 2019;13(3):383-390 